\title{
Brownian Motion: the Quantum Perspective
}

\author{
Bassano Vacchini \\ Dipartimento di Fisica dell'Università di Milano and Istituto Nazionale di Fisica Nucleare, Sezione \\ di Milano, Via Celoria 16, I-20133 Milano \\ Reprint requests to Dr. B. V. E-mail: bassano.vacchini@mi.infn.it
}

Z. Naturforsch. 56 a, 230-233 (2001); received February 6, 2001

Presented at the 3rd Workshop on Mysteries, Puzzles and Paradoxes in Quantum Mechanics, Gargnano, Italy, September 17 - 23, 2000.

\begin{abstract}
We briefly go through the problem of the quantum description of Brownian motion, concentrating on recent results about the connection between the dynamics of the particle and dynamic structure factor of the medium. - 05.40.Jc, 05.30.Fk, 05.30.Jp, 03.65.Yz
\end{abstract}

Key words: Quantum Brownian Motion; Dynamic Structure Factor; Fokker-Planck; Lindblad.

Early in the nineteenth century, the biologist Robert Brown wrote a paper on random motion of a large particle immersed in a fluid [1], a paper which received wide attention, leading to the phenomenon being named after him. Despite the raised interest, the first correct theoretical description of the phenomenon, which had previously been tentatively explained in terms of irregular heating due to incident light or some kind of electrical forces, was only given after almost a century on the basis of the so-called random walk problem in a number of papers (beginning 1905 [2]) by Albert Einstein, who was looking for a way to confirm the atomic nature of matter. These studies led to a relation between the macroscopic diffusion coefficient and the atomic properties of matter, which links the irreversible nature of the phenomenon to the mechanism of molecular fluctuations, thus providing the first example of a fluctuation-dissipation relation, the key point being the connection between the dynamics of the Brownian particle and statistical mechanics properties of the fluid.

The description of the phenomenon at classical level relies on differential equations of the FokkerPlanck type for the different distribution functions [3], and in particular the velocity distribution of the Brownian particle of mass $M$ obeys an equation of the form

$$
\frac{\partial p(v, t)}{\partial t}=\eta \frac{\partial}{\partial v}[v p(v, t)]+D_{v} \frac{\partial^{2}}{\partial v^{2}} p(v, t),
$$

where $\eta$ is the friction constant, and the diffusion coefficient $D_{v}=\eta / M \beta$ involves $\beta=\left(k_{\mathrm{B}} T\right)^{-1}$, the (inverse) energy scale set by the temperature $T$ and Boltzmann's constant $k_{\mathrm{B}}$. It is however not immediately obvious how to tackle the problem in the quantum case, since due to dissipation and irreversibility one is not dealing with a Hamiltonian system and therefore the standard quantization procedure fails. At this point one can either resort to new quantization schemes, which might lead to meaningful results for this particular type of problems [4], or more consistently consider a system reservoir approach inside a thorough quantum mechanical formalism [5], aiming at the description of the subdynamics of the Brownian particle with respect to the fluid. A first naive guess of the possible result may be obtained applying the correspondence principle to (1), written however in terms of momenta, substituting to the classical variables the operators $\hat{x}$ and $\hat{p}$ acting in the usual way in the Hilbert space of the particle, thus obtaining in the Schrödinger picture a structure of the form

$$
\frac{\mathrm{d} \hat{\varrho}}{\mathrm{d} t}=-\frac{i}{\hbar}[\hat{H}, \hat{\varrho}]-\frac{i}{\hbar} \gamma[\hat{x},\{\hat{p}, \hat{\varrho}\}]-\frac{D_{p p}}{\hbar^{2}}[\hat{x},[\hat{x}, \hat{\varrho}]],
$$

where for the sake of simplicity we have here restricted ourselves to a one dimensional notation. This somehow expected result was in fact obtained in a pioneering work by Caldeira and Leggett [6], using a Feynman path integral formalism for the description 
of a particle coupled to a bath of harmonic oscillators. In particular their result takes the form

$$
\begin{aligned}
\frac{\mathrm{d} \varrho}{\mathrm{d} t}= & -\frac{i}{\hbar}[\hat{H}, \hat{\varrho}]-\frac{i}{\hbar} \gamma[\hat{x},\{\hat{p}, \hat{\varrho}\}] \\
& -\frac{1}{\hbar^{2}} \frac{2 M \gamma}{\beta}[\hat{x},[\hat{x}, \hat{\varrho}]],
\end{aligned}
$$

where $\gamma$ is a phenomenological parameter linked to the bath properties. It was however later realized that this master equation does not always preserve positivity of the statistical operator $[7,8]$ : in fact it does not have a completely positive structure $[9,10]$, complete positivity being for this class of systems equivalent to positivity [11]. A completely positive time evolution is in fact driven by a master equation having a Lindblad structure [12]

$\frac{\mathrm{d} \varrho}{\mathrm{d} t}=-\frac{i}{\hbar}[\hat{H}, \varrho \varrho]-\frac{1}{2 \hbar} \sum_{i}\left\{\hat{V}_{i}^{\dagger} \hat{V}_{i}, \hat{\varrho}\right\}+\frac{1}{\hbar} \sum_{i} \hat{V}_{i} \hat{\varrho} \hat{V}_{i}^{\dagger}$,

which in the case of Brownian motion, in order to give a friction force proportional to velocity, takes the following form, with contributions at most bilinear in the operators $\hat{x}$ and $\hat{p}$, according to a choice of generators of the form $\hat{V}=a \hat{x}+b \hat{p}[13]$

$$
\begin{aligned}
\frac{\mathrm{d} \hat{\varrho}}{\mathrm{d} t}= & -\frac{i}{\hbar}[\hat{H}, \hat{\varrho}]-\frac{i}{\hbar} \mu[\hat{\varrho},\{\hat{x}, \hat{p}\}] \\
& -\frac{i}{\hbar} \gamma[\hat{x},\{\hat{p}, \hat{\varrho}\}]-\frac{D_{x x}}{\hbar^{2}}[\hat{p},[\hat{p}, \hat{\varrho}]] \\
& -\frac{D_{p p}}{\hbar^{2}}[\hat{x},[\hat{x}, \hat{\varrho}]]+2 \frac{D_{x p}}{\hbar^{2}}[\hat{p},[\hat{x}, \hat{\varrho}]],
\end{aligned}
$$

the coefficients being constrained by the following requirements:

$$
\begin{aligned}
& D_{p p}>0, D_{x x}>0, \\
& D_{x x} D_{p p}-D_{x p}^{2} \geq(\gamma \hbar / 2)^{2},
\end{aligned}
$$

which ensure complete positivity. Equation (3) is not of Lindblad form, since $D_{x x}=0$, and quite a lot of work has been done in order to cope with this difficulty [14], both at fundamental and phenomenological level, usually considering an harmonically bound particle interacting with a bath of harmonic oscillators, leading to a typical correction of the form

$$
D_{x x}=\chi \frac{\beta \hbar^{2}}{M} \gamma,
$$

with $\chi \geq 1 / 8$, corresponding to a higher order correction in the high temperature limit actually considered by Caldeira and Leggett.

Most recently a microphysical scattering theory derivation of a master equation driving the subdynamics of a test particle interacting through collisions with a fluid has been considered and applied to the specific case of the quantum description of Brownian motion [15 - 17]. Let us note that the particular case of Brownian motion bears some peculiar features with respect to the more general dissipative systems described in [13], as stressed in [14, 18] and often neglected in the literature. The main result of [17] is the following structure of the master equation for the subdynamics of the test particle immersed in a homogeneous fluid of not necessarily free particles:

$$
\begin{aligned}
\frac{\mathrm{d} \varrho}{\mathrm{d} t}=-\frac{i}{\hbar}\left[\hat{H}_{0}, \hat{\varrho}\right]+\frac{2 \pi}{\hbar}(2 \pi \hbar)^{3} n & \cdot \int \mathrm{d}^{3} q|\tilde{t}(q)|^{2}\left[L(q, \hat{p}, \hat{x}) \hat{\varrho} L^{\dagger}(q, \hat{p}, \hat{x})\right. \\
& \left.-\frac{1}{2}\left\{L^{\dagger}(q, \hat{p}, \hat{x}) L(q, \hat{p}, \hat{x}), \hat{\varrho}\right\}\right],
\end{aligned}
$$

where $\hat{H}_{0}$ is the Hamiltonian of the free particle, $\hat{\varrho}$ its statistical operator, $n$ the particle density in the fluid, $\tilde{t}(q)$ the Fourier transform of the T matrix describing two-particle collisions evaluated for the transferred momentum $q$, and

$$
L(q, \hat{p}, \hat{x})=e^{\frac{i}{\hbar} q \cdot \hat{x}} \sqrt{S(q, \hat{p})},
$$

with $S(q, \hat{p})$ the dynamic structure function of the medium. Expression (7), with its typical Lindblad structure, can be obtained under a suitable interplay between energy dependence of the dynamic structure factor and quasi-diagonality of the matrix elements of $\hat{\varrho}$ in the momentum representation, valid if one works on a time scale $\tau$ over which the subdynamics of the particle is suitably slow. The dynamic structure factor, usually given in terms of energy and momentum transfer ( $E$ being the energy transfer corresponding to scattering from state $p$ to state $p^{\prime}=p+q$ ), is the 
B. Vacchini - Brownian Motion: the Quantum Perspective

Fourier transform of the two-point density correlation function of the medium [19]

$$
\begin{array}{r}
S(q, E)=\frac{1}{2 \pi \hbar} \frac{1}{N} \int \mathrm{d} t \int \mathrm{d}^{3} x e^{\frac{i}{\hbar}(E t-q \cdot x)} \\
\cdot \int \mathrm{d}^{3} y\langle N(y) N(x+y, t)\rangle
\end{array}
$$

and can be measured in terms of scattering experiments, being strictly connected to the inelastic scattering cross-section of a microscopic probe of a macroscopic sample [20]. Expression (8) depends on the statistical mechanics properties of the system and in particular on its equilibrium fluctuations, so that according to (7) the dynamics of the particle depends on the density fluctuations of the medium, thus putting into major evidence through the appearance of the quantum correlation function (8) one of the main ideas of the classical approach of Einstein to the problem. To proceed further we have to give a specific example of a dynamic structure factor, and we consider the case of a free gas. To restrict to Brownian motion one takes a particle of mass $M$ much heavier than the mass $m$ of the particles making up the gas. In the case of Boltzmann particles this leads to [16]

$$
\begin{aligned}
& \frac{\mathrm{d} \varrho}{\mathrm{d} t}=-\frac{i}{\hbar}\left[\hat{H}_{0}, \hat{\varrho}\right]+z \frac{4 \pi^{2} m^{2}}{\beta \hbar} \int \mathrm{d}^{3} q \frac{|\tilde{t}(q)|^{2}}{q} e^{-\frac{\beta}{8 m} q^{2}}(9) \\
& \cdot\left[e^{\frac{i}{\hbar} q \cdot \hat{x}} e^{-\frac{\beta}{4 M} q \cdot \hat{p}} \hat{\varrho} e^{-\frac{\beta}{4 M} q \cdot \hat{p}} e^{-\frac{i}{\hbar} q \cdot \hat{x}}-\frac{1}{2}\left\{e^{-\frac{\beta}{2 M} q \cdot \hat{p}}, \hat{\varrho}\right\}\right],
\end{aligned}
$$

where $z$ is the fugacity of the gas, and in the limit of small momentum transfer or long wavelength fluctuations, in order to recover the analogoue of the FokkerPlanck equation, with a friction force proportional to the velocity, one comes to

$$
\begin{array}{r}
\frac{\mathrm{d} \varrho}{\mathrm{d} t}=-\frac{i}{\hbar}\left[\hat{H}_{0}, \hat{\varrho}\right]-z \sum_{i=1}^{3}\left\{\frac{D_{p p}}{\hbar^{2}}\left[\hat{x}_{i},\left[\hat{x}_{i}, \hat{\varrho}\right]\right]\right. \\
\left.+\frac{D_{x x}}{\hbar^{2}}\left[\hat{p}_{i},\left[\hat{p}_{i}, \hat{\varrho}\right]\right]+\frac{i}{\hbar} \gamma\left[\hat{x}_{i},\left\{\hat{p}_{i}, \hat{\varrho}\right\}\right]\right\},
\end{array}
$$

where the coefficients are fixed at microphysical level by the expressions

$$
\begin{aligned}
& D_{p p}=\frac{2}{3} \frac{\pi^{2} m^{2}}{\beta \hbar} \int \mathrm{d}^{3} q|\tilde{t}(q)|^{2} q e^{-\frac{\beta}{8 m} q^{2}}, \\
& D_{x x}=(\beta \hbar / 4 M)^{2} D_{p p}, \quad \gamma=(\beta / 2 M) D_{p p} .
\end{aligned}
$$

Equation (10) corresponds to a Lindblad structure with $\chi=1 / 8$, i. e., the minimal modification to (3) which copes with complete positivity. In particular this implies that (10) can be written in terms of a single generator for each Cartesian direction, as one can see introducing the operators $\hat{a}_{i}=\frac{\sqrt{2}}{\lambda_{M}}\left(\hat{x}_{i}+\frac{i}{\hbar} \frac{\lambda_{M}^{2}}{4} \hat{p}_{i}\right)$, with $\lambda_{M}=\sqrt{\hbar^{2} / M k T}$ the thermal wavelength of the Brownian particle (resulting as usual $\left[\hat{a}_{i}, \hat{a}_{j}^{\dagger}\right]=\delta_{i j}$ ), thus coming to

$$
\begin{aligned}
\frac{\mathrm{d} \varrho}{\mathrm{d} t}= & -\frac{i}{\hbar}\left[\hat{H}_{0}, \hat{\varrho}\right]-z \frac{D_{p p}}{\hbar^{2}} \frac{\lambda_{M}^{2}}{4} \sum_{i=1}^{3} \frac{i}{\hbar}\left[\left\{\hat{x}_{i}, \hat{p}_{i}\right\}, \hat{\varrho}\right] \\
& +\frac{D_{p p}}{\hbar^{2}} \lambda_{M}^{2} \sum_{i=1}^{3}\left[\hat{a}_{i} \hat{\varrho} \hat{a}_{i}^{\dagger}-\frac{1}{2}\left\{\hat{a}_{i}^{\dagger} \hat{a}_{i}, \hat{\varrho}\right\}\right],
\end{aligned}
$$

where the single generator structure is due to the fact that the coefficients in (11) are actually linked by $D_{p p} D_{x x}=\hbar^{2} \gamma^{2} / 4$. It is now also possible to consider the extension to quantum statistics, thus obtaining a master equation describing the motion of a Brownian particle in a Bose or Fermi gas. To do this one has to calculate the dynamic structure factor of a free Bose or Fermi gas, taking then the Brownian limit in which the ratio between the masses is much smaller than one and the limit of small momentum transfer. The result one obtains, when expressed in terms of the fugacity $z$ of the gas is in fact particularly simple,

$$
\begin{aligned}
\frac{\mathrm{d} \varrho}{\mathrm{d} t}= & -\frac{i}{\hbar}\left[\hat{H}_{0}, \hat{\varrho}\right]-\frac{z}{1 \mp z} \sum_{i=1}^{3}\left\{\frac{D_{p p}}{\hbar^{2}}\left[\hat{x}_{i},\left[\hat{x}_{i}, \hat{\varrho}\right]\right]\right. \\
& \left.+\frac{D_{x x}}{\hbar^{2}}\left[\hat{p}_{i},\left[\hat{p}_{i}, \hat{\varrho}\right]\right]+\frac{i}{\hbar} \gamma\left[\hat{x}_{i},\left\{\hat{p}_{i}, \hat{\varrho}\right\}\right]\right\},(13)
\end{aligned}
$$

the operator structure is preserved, but the dependence on the fugacity is through $z /(1 \mp z)$ rather than through $z$, thus recovering for small fugacity the Boltzmann case (10). From (13) one also obtains a definite expression for the ratio between the friction 
coefficient for the Maxwell Boltzmann and Bose or Fermi statistics

$$
\frac{\gamma_{\mathrm{MB}}}{\gamma_{\mathrm{B} / \mathrm{F}}}=1 \mp z,
$$

showing that friction is enhanced or suppressed according to statistics.

[1] R. Brown, Philosophical Magazine N. S. 4, 161 (1828).

[2] A. Einstein, Ann. Phys. (Leipzig) 17, 549 (1905).

[3] H. Risken, The Fokker-Planck Equation 2nd ed., Springer-Verlag, Berlin 1989.

[4] H. Dekker, Phys. Rep. 80, 1 (1981).

[5] H. Spohn, Rev. Mod. Phys. 53, 569 (1980).

[6] A. O. Caldeira and A. J. Leggett, Physica A 121, 587 (1983).

[7] V. Ambegaokar, Ber. Bunsenges Phys. Chem. 95, 400 (1991).

[8] P. Pechukas, in Large Scale Molecular Systems, edited by W. Gans, A. Blumen, and A. Amann, NATO ASI Series, Plenum Press, New York 1991, Vol. 258, p. 123.

[9] G. Lindblad, Rep. Math. Phys. 10, 393 (1976).

[10] A. Barchielli, Nuovo Cimento 74B, 113 (1983).

\section{Acknowledgement}

The author would like to thank Prof. L. Lanz for useful discussions. This work was supported by MURST under Cofinanziamento and Progetto Giovani.

[11] P. Talkner, Ann. Phys. (New York) 167, 390 (1986).

[12] G. Lindblad, Commun. Math. Phys. 48, 119 (1976).

[13] A. Săndulescu and H. Scutaru, Ann. Phys. (New York) 173, 277 (1987).

[14] D. Kohen, C. C. Marston and D. J. Tannor, J. Chem. Phys. 107, 5236 (1997).

[15] L. Lanz and B. Vacchini, Int. J. Theor. Phys. 36, 67 (1997).

[16] B. Vacchini, Phys. Rev. Lett. 84, 1374 (2000).

[17] B. Vacchini, quant-ph/0012129, to appear in Phys. Rev. E.

[18] B. Vacchini, to appear in Phys. Rev. Lett.

[19] S. W. Lovesey, Theory of Neutron Scattering from Condensed Matter, Clarendon Press, Oxford 1984.

[20] L. van Hove, Phys. Rev. 95, 249 (1954). 
Nachdruck - auch auszugsweise - nur mit schriftlicher Genehmigung des Verlages gestattet Verantwortlich für den Inhalt: A. KLEMM

Satz und Druck: Konrad Triltsch, Print und digitale Medien GmbH, Ochsenfurt/Hohestadt 